\title{
Smooth Haired Standard Dachshund
}

National Cancer Institute

\section{Source}

National Cancer Institute. Smooth Haired Standard Dachshund. NCI Thesaurus. Code C53903.

The Smooth Haired Standard Dachshund is a smooth-haired variety of dachshund with a standard height up to 9 inches. 